\title{
Check-list das Erythroxylaceae no estado de Mato Grosso do Sul, Brasil
}

\author{
Maria Iracema Bezerra Loiola \& Luciana Silva Cordeiro
}

Universidade Federal do Ceará, Herbário EAC, Departamento de Biologia, Campus do Pici, Bloco 906, CEP 60455-760, Fortaleza, Ceará Brasil. iloiola@ufc.com.br

Recebido em 27.IX 2014

Aceito em 06.V.2016

DOI 10.21826/2446-8231201873s201

RESUMO - Para o estado do Mato Grosso do Sul foram registradas 12 espécies, das quais Erythroxylum subrotundum A.St.-Hil. constitui nova ocorrência e E. patentissimum O.E.Schulz tem distribuição restrita a esse estado.

Palavras-chave: distribuição geográfica, diversidade, flora, neotrópico

ABSTRACT - Checklist of Erythroxylaceae from Mato Grosso do Sul state, Brazil. For the Mato Grosso do Sul state was found 12 species, of which Erythroxylum subrotundum A.St.-Hil. Is new record and E. patentissimum O.E.Schulz is endemic.

Keywords: diversity, flora, geographic distribution, neotropic

\section{INTRODUÇÃO}

Erythroxylaceae Kunth compreende quatro gêneros e aproximadamente 250 espécies (Mabberley 1990), estando representada nos trópicos apenas por Erythroxylum P. Br. (240 spp.). Os gêneros Aneulophus Benth. (duas spp.), Nectaropetalum Engler (seis spp.) e Pinacopodium Exell \& Mendonça (duas spp.) têm distribuição exclusiva na África (Daly 2004).

$\mathrm{Na}$ região Neotropical ocorrem 187 espécies de Erythroxylum (Plowman \& Hensold 2004), sendo o Brasil um dos centros de diversificação e endemismo deste gênero (Plowman \& Berry 1999). Em território brasileiro foram registradas 127 espécies, das quais 83 têm distribuição restrita (Loiola \& Costa-Lima 2015). Ainda segundo estes autores, as espécies de Erythroxylum têm ampla distribuição, sendo encontradas em diferentes domínios fitogeográficos como Amazônia, Caatinga, Cerrado e Mata Atlântica, e nos mais diversos tipos de vegetação, dentre estes Caatinga (stricto sensu), Campinarana, Campo Rupestre, Carrasco, Cerrado (lato sensu), Floresta Ciliar e/ou de Galeria, Floresta de Terra-Firme, Floresta Estacional Decidual, Floresta Estacional Semidecidual, Floresta Ombrófila (=Floresta Pluvial), Restinga e Savana Amazônica.

Os representantes de Erythroxylum compartilham as seguintes características: hábito variando de subarbustos a árvores de pequeno a médio porte (3-10 $\mathrm{m}$ de altura), catafilos geralmente semelhantes às estípulas; folhas sempre alternas e inteiras, com estípulas intrapeciolares, estriadonervadas ou não, frequentemente 2 a 3 -setulosas no ápice; inflorescências constituídas por uma a várias flores, em geral menores que um $\mathrm{cm}$, actinomorfas, diclamídeas, pentâmeras, hermafroditas e heterostílicas; estames dez, com os filetes concrescidos na base formando um pequeno tubo; ovário súpero, tricarpelar, com três lóculos (dos quais dois são vazios e o terceiro aloja um único óvulo) e fruto do tipo drupa.

Entre os tratamentos infragenéricos destacam-se os elaborados por De Candolle (1824), Martius (1840, 1843) e Peyritsch (1878), por focarem as espécies brasileiras. Já Schulz (1907), baseado principalmente nos caracteres morfológicos das estípulas e catafilos, reconheceu 19 seções para o gênero; 10 abrangendo as espécies asiáticas e africanas e nove as americanas, reconhecendo um total de 193 espécies. O primeiro grupo, caracterizado pelas estípulas e catafilos com estrias muito evidentes $\mathrm{e}$, às vezes ainda, densamente vilosa com margem fimbriolada foram incluídas as seções Pogonophorum, Rhabdophyllum e Macrocalyx. No segundo, que abrangia as espécies com estrias pouco evidentes ou ausentes, com margem levemente ou não fimbriolada foram agrupadas as seções: Leptogramme, Heterogyne, Archerythroxylum, Megalophyllum, Mastigophorum, Microphyllum, Melanocladus, Gonocladus, Sethia, Lagynocarpus, Coelocarpus, Eurysepalum, Venelia, Pachylobus, Schistophyllum e Oxystigma. Merece destacar que esta circunscrição é ainda hoje usada pelos estudiosos deste gênero.

No Brasil, entre os autores que mais contribuíram para o entendimento do gênero e que focaram seus estudos na descrição de novas espécies e distribuição destas em território brasileiro destacam-se Plowman, que em 1983 descreveu novas espécies de Erythroxylum para o Brasil e Venezuela; em 1984, reconheceu novas espécies para a Amazônia brasileira; em 1986, descreveu quatro novas 
espécies para o nordeste do Brasil e em 1987, propôs dez novas espécies para o estado da Bahia. Já Amaral Jr. desenvolveu vários estudos em diferentes regiões do Brasil, merecendo destaque o trabalho de 1973, onde apresentou o estudo taxonômico das espécies de Erythroxylum ocorrentes no município de Botucatu, São Paulo, registrando dez taxa; o de 1976, onde descreveu Erythroxylum campinense para a região Amazônica e o de 1980, que registrou dez espécies e uma variedade de Erythroxylum para Santa Catarina, reconhecendo ainda uma nova espécie para o referido estado (E. catharinense).

Mais recentemente, Loiola elaborou uma série de estudos focando os representantes de Erythroxylum principalmente no nordeste brasileiro. As principais contribuições desta autora foram: a "Revisão taxonômica dos representantes de Erythroxylum sect. Rhabdophyllum para a região Neotropical", onde reconheceu 51 espécies (Loiola 2001), a descrição de novas espécies de Erythroxylum para as regiões nordeste e sudeste do Brasil (Loiola 2013, Loiola \& Sales 2008, 2012, Loiola \& Cordeiro 2014), a elaboração de algumas floras locais (Loiola 2004, Loiola et al. 1998, Loiola \& Gomes 2009) ou estaduais (Loiola et al. 2007, Loiola 2008) e a sinonimização e lectotipificação de alguns taxa do gênero (Loiola et al. 2015). Merece destacar ainda uma série de estudos realizados por Costa-Lima especialmente com os representantes de Erythroxylum ocorrentes na floresta Atlântica. Esse autor propôs a descrição de novas espécies (Costa-Lima et al. 2013a, 2014a, 2015a) e tem contribuído para uma melhor compreensão sobre a taxonomia e distribuição deste gênero (Costa-Lima et al. 2013b, 2014b, 2015b). Destaca-se ainda que estes autores vêm trabalhando em colaboração já há algum tempo e elaboraram a lista dos representantes de Erythroxylum ocorrentes em território brasileiro, reconhecendo 127 espécies (Loiola \& Costa-Lima 2015).

Embora seja um grupo bem representado na flora brasileira e tenha sido objeto de estudo de diferentes pesquisadores, os trabalhos sobre os representantes de Erythroxylum no Brasil ainda são incipientes, uma vez que atualmente, ainda faltam informações sobre o número de espécies que ocorrem em vários estados, especialmente nas regiões Norte e Nordeste do país.

Com o intuito de suprir parte desta lacuna de conhecimento, este trabalho objetivou registrar as espécies de Erythroxylum ocorrentes no Mato Grosso do Sul, visando conhecer a diversidade da flora do referido estado e contribuir com informações sobre a distribuição geográfica dessas espécies.

\section{MATERIAL E MÉTODOS}

Para a elaboração da lista e distribuição geográfica das espécies ocorrentes em Mato Grosso do Sul foram consultadas bibliografias especializadas (Peyritsch 1878, Dubs 1998, Barbosa \& Amaral Jr. 2001, Loiola 2001, Plowman \& Hensold 2004) e sítios na internet (Ratter et al. 2013, CRIA 2012, Loiola \& Costa Lima 2015).
Os nomes dos autores estão abreviados de acordo com Brummitt \& Powell (1992). A lista contém os nomes científicos e populares, citação de um material de referência (voucher), informações sobre o hábito, distribuição geográfica, domínio fitogeográfico e tipo de vegetação preferencial de cada espécie. Tendo em vista que a maioria das coleções analisadas para o Mato Grosso do Sul não indica o tipo de vegetação que as amostras foram coletadas, optou-se em fazer comentários mais gerais sobre a distribuição das espécies.

A classificação das espécies por hábito foi feita com base em Cain \& Castro (1959) e Whittaker (1975), como descrito seguir: a) árvore - planta lenhosa com no mínimo $3 \mathrm{~m}$ de altura; b) arbusto - planta lenhosa com no mínimo $1 \mathrm{~m}$ e no máximo $3 \mathrm{~m}$ de altura e c) subarbusto - planta com no máximo $1 \mathrm{~m}$ de altura, caule principal lenhoso com ramificações secundárias herbáceas.

\section{RESULTADOS E DISCUSSÃO}

Para o Mato Grosso do Sul foram registradas 12 espécies de Erythroxylum em diferentes municípios (Fig. 1). E. subrotundum A.St-Hil. constitui um novo registro e $E$. patentissimum O.E.Schulz uma espécie com distribuição exclusiva para esse estado, tendo registro apenas no domínio fitogeográfico pantanal.

Quanto à forma de crescimento, verificou-se que a maioria das espécies apresenta hábito variável; no entanto, são predominantemente arbustivas. Do total, 12 espécies (100\%) apresentaram hábito arbustivo, nove (75\%) arbóreo e cinco $(41,66 \%)$ subarbustivo.

Verificou-se que a maioria das espécies tem distribuição em dois ou mais países da América do Sul e em dois ou mais domínios fitogeográficos (Quadro 1). Erythroxylum citrifolium A.St.-Hil. destacou-se das demais por apresentar ampla distribuição na região Neotropical, com registro desde o México até o sul do Brasil, e nos mais diversos tipos de vegetação (Loiola 2001). Por outro lado, E. engleri O.E.Schulz e E. subrotundum A.St-Hil. ocorrem exclusivamente no Brasil. Já E. tortuosum Mart. com ocorrência confirmada para a Bolívia e Brasil, foi registrada apenas em vegetação de cerrado em território brasileiro.

Os estudos realizados em outros estados brasileiros mostram que o número, a relação das espécies e a preferência por ambiente são muito variáveis. Amaral Jr. (1973) apresentou o estudo taxonômico das espécies de Erythroxylum ocorrentes município de Botucatu/ São Paulo, no qual registrou dez táxons desenvolvendo preferencialmente em ambientes de cerrados e matas. Loiola et al. (2007) reconheceram 13 espécies de Erythroxylum para o estado da Paraíba, ocorrendo preferencialmente em ambientes úmidos da floresta Atlântica. Já Cordeiro (2010), realizou um estudo sobre as espécies de Erythroxylum para o Ceará, onde registrou 26 táxons habitando principalmente em ambientes secos como carrasco e caatinga. Costa-Lima et al. (2013) registraram 17 espécies para Sergipe, das quais 14 ocorrem nas formações vegetais da mata Atlântica, 


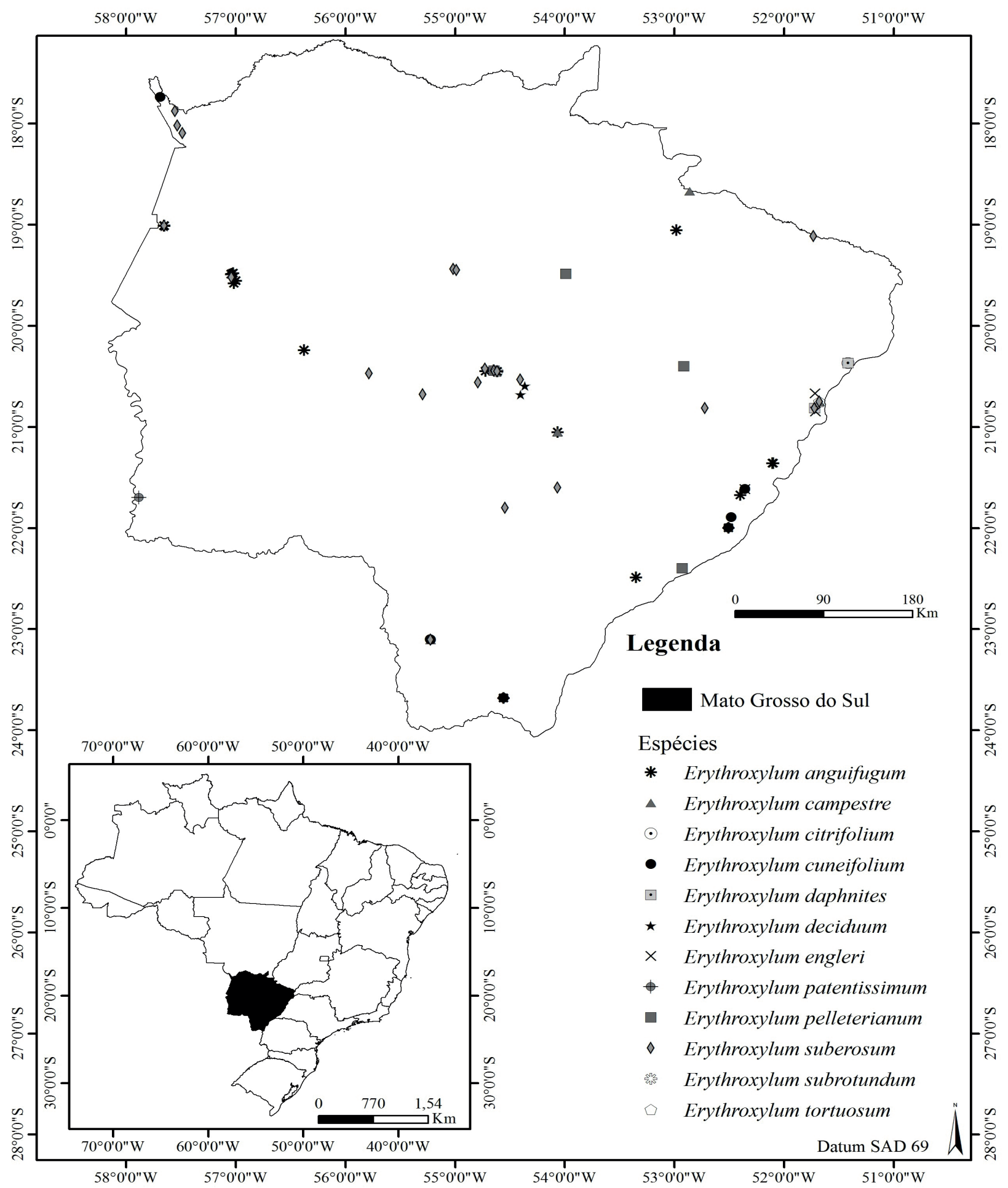

Fig. 1. Mapa de localização do estado do Mato Grosso de Sul - Brasil, evidenciando os locais de coleta das espécies de Erythroxylum registradas para o Mato Grosso do Sul, Brasil.

como restingas, tabuleiros litorâneos, florestas estacionais e florestas ombrófilas.

Merece destacar que o único trabalho que tratou os representantes de Erythroxylum na área de abrangência dos estados do Mato Grosso e Mato Grosso do Sul foi desenvolvido por Dubs (1998), que na obra intitulada "Prodromus Florae Matogrossensi" registrou 23 espécies de
Erythroxylum. Dentre essas, nove foram registradas no Mato Grosso do Sul, sendo uma (E. patentissimum O.E.Schulz) com distribuição exclusiva nesse estado; já para o estado do Mato Grosso foram identificadas 22 espécies, sendo 14 com distribuição restrita. Das 23 espécies listadas, apenas nove foram registradas em ambos os estados.

Ao comparar os dados obtidos nos trabalhos supracitados 
Quadro 1. Lista das espécies de Erythroxylum registradas no estado de Mato Grosso do Sul com os respectivos vouchers, formas de crescimento, distribuição geográfica e domínio fitogeográfico. Formas de crescimento $=\mathrm{FC}(\mathrm{Ar}=$ árvore; $\mathrm{Arb}=$ arbusto; $\mathrm{Subar}=$ subarbusto $)$. Distribuição geográfica $(\mathrm{AL}=$ Alagoas, $\mathrm{AM}=$ Amazonas, $\mathrm{AP}=$ Amapá, $\mathrm{BA}=$ Bahia, $\mathrm{CE}=$ Ceará, $\mathrm{DF}=$ Distrito Federal, $\mathrm{ES}=$ Espírito $\mathrm{Santos}, \mathrm{GO}=\mathrm{Goiás}$, $\mathrm{MA}=$ Maranhão, $\mathrm{MG}=$ Minas Gerais, $\mathrm{MT}=$ Mato Grosso, $\mathrm{MS}=$ Mato Grosso do Sul, $\mathrm{PA}=$ Pará, $\mathrm{PB}=$ Paraíba, $\mathrm{PE}=\mathrm{Pernambuco}, \mathrm{PR}=\mathrm{Paraná}$, $\mathrm{RJ}=$ Rio de Janeiro, $\mathrm{RO}=$ Rondônia, $\mathrm{RR}=$ Roraima, $\mathrm{RS}=$ Rio Grande do Sul, $\mathrm{SC}=$ Santa Catarina, $\mathrm{SP}=$ São Paulo, $\mathrm{TO}=$ Tocantins $). *$ Nova ocorrência para Mato Grosso do Sul.

\begin{tabular}{|c|c|c|c|c|}
\hline Espécies & Voucher & FC & Distribuição geográfica & Domínio fitogeográfico \\
\hline E. anguifugum Mart. & $\begin{array}{c}\text { Maracaju: Faz. Santo Antonio, } \\
\text { Sucre } 10490(\mathrm{~F})\end{array}$ & Arb, Arv & $\begin{array}{c}\text { Espécie sulamericana, registrada } \\
\text { na Bolívia, Brasil, Paraguai e Peru. } \\
\text { Brasil (AC, AM, GO, MA, } \\
\text { MG, MS, MT, PA, PR, RJ, RO, } \\
\text { RR, SP, TO) }\end{array}$ & Amazônia, Cerrado \\
\hline E. campestre A.St.-Hil. & $\begin{array}{c}\text { Iguatemi: MS-295, } 15 \mathrm{~km} \mathrm{~L} \\
\text { Iguatemi, Hatschbach et al. } 58595 \\
\text { (BOTU, ESA, F) }\end{array}$ & Subar & $\begin{array}{c}\text { Espécie sulamericana, com } \\
\text { ocorrência na Bolívia, Brasil, e } \\
\text { Paraguai. } \\
\text { Brasil (BA, CE, DF, GO, MA, } \\
\text { MG, MS, MT, PR, RJ, RO, SP) }\end{array}$ & Cerrado, Mata Atlântica \\
\hline E. citrifolium A.St.-Hil. & $\begin{array}{l}\text { Selviria: Faz. Do Sr. Cacildo } \\
\text { Arantes, Martins, F.R., et al. } 188 \\
\text { (CGMS) } \\
\text { Citada por Barbosa \& Amaral Jr. } \\
\text { (2001) para Mato Grosso do Sul }\end{array}$ & $\begin{array}{l}\text { Subar, Arb, } \\
\text { Arv }\end{array}$ & $\begin{array}{l}\text { Espécie Neotropical, com } \\
\text { ampla distribuição desde o } \\
\text { México até o sul do Brasil. } \\
\text { Brasil (AL, AP, AM, BA, DF, ES, } \\
\text { GO, MA, MG, MS, MT, PA, PB, } \\
\text { PE, RJ, RO, RR, SE, SP, TO) }\end{array}$ & $\begin{array}{l}\text { Amazônia, Cerrado, } \\
\text { Mata Atlântica }\end{array}$ \\
\hline $\begin{array}{l}\text { E. cuneifolium (Mart.) } \\
\text { O.E.Schulz }\end{array}$ & $\begin{array}{c}\text { Amambái: Hatschbach } 45975 \\
\text { (MBM, Z) }\end{array}$ & Arb, Arv & $\begin{array}{c}\text { Espécie sulamericana, registra- } \\
\text { da na Argentina, Brasil, Bolívia } \\
\text { e Paraguai. } \\
\text { Brasil (GO, MG, MS, MT, PR, } \\
\text { RJ, RS, SC, SP) }\end{array}$ & Cerrado, Mata Atlântica \\
\hline E. daphnites Mart. & $\begin{array}{c}\text { Selvíria: Faz. Santa Maria, Fonan } \\
\text { et al. } 137 \text { (HRCB) }\end{array}$ & Arb, Arv & $\begin{array}{c}\text { Espécie sulamericana, com } \\
\text { ocorrência na Argentina, Bolí- } \\
\text { via, Brasil e Paraguai. } \\
\text { Brasil (GO, MG, MS, MT, PR, } \\
\text { RS, RJ, SC, SP) }\end{array}$ & $\begin{array}{l}\text { Amazônia, Caatinga, } \\
\text { Cerrado, Mata Atlântica }\end{array}$ \\
\hline E. deciduum A.St.-Hil. & $\begin{array}{l}\text { Campo Grande: Reserva SEMA, } \\
\text { Conceição } 2173 \text { (UFMS, PEUFR) }\end{array}$ & $\begin{array}{l}\text { Sub, Arb, } \\
\text { Arv }\end{array}$ & $\begin{array}{c}\text { Espécie sulamericana, com } \\
\text { registros na Argentina, Brasil, } \\
\text { Paraguai e Peru. } \\
\text { Brasil (BA, DF, GO, MA, MG, } \\
\text { MS, MT, PA, PR, RJ, RS, SC, SP) }\end{array}$ & $\begin{array}{l}\text { Amazônia, Cerrado, } \\
\text { Mata Atlântica }\end{array}$ \\
\hline E. engleri O.E.Schulz & $\begin{array}{l}\text { Três Lagoas: cerca de } 8 \mathrm{~km} \text { da } \\
\text { MS-395, Dias et al. } 45 \text { (BOTU) }\end{array}$ & Arb, Arv & $\begin{array}{l}\text { Espécie restrita ao Brasil (DF, } \\
\text { GO, MA, MG, MS, MT, TO) }\end{array}$ & $\begin{array}{l}\text { Amazônia, Caatinga, } \\
\text { Cerrado }\end{array}$ \\
\hline E. patentissimum O.E.Schulz & $\begin{array}{l}\text { Porto Murtinho: } 30 \text { km leste de } \\
\text { Porto Murtinho, J. da Silva Costa } \\
160 \text { (F, RB ) }\end{array}$ & Arb & & Não indicado \\
\hline E. pelleterianum A.St.-Hil. & $\begin{array}{l}\text { Selvíria: Faz. Santa Maria, Mar- } \\
\text { tins et al. } 63 \text { (HRCB) }\end{array}$ & Arb, Arv & $\begin{array}{c}\text { Espécie sulamericana, com } \\
\text { ocorrência na Argentina, Bolí- } \\
\text { via, Brasil, Paraguai e Uruguai. } \\
\text { Brasil (BA, GO, MG, MS, MT, } \\
\text { PR, RJ, RS, SP) }\end{array}$ & Caatinga, Cerrado \\
\hline E. suberosum A.St.-Hil. & $\begin{array}{c}\text { Amambái: road Cel. Sapucaia, } \\
\text { Hatschbach } 48416 \text { (F, MBM) }\end{array}$ & $\begin{array}{l}\text { Sub, Arb, } \\
\text { Arv }\end{array}$ & $\begin{array}{c}\text { Espécie sulamericana, registrada } \\
\text { na Bolívia, Brasil, Guiana Fran- } \\
\text { cesa, Paraguai e Venezuela. } \\
\text { Brasil (AC, AL, AP, AM, BA, } \\
\text { CE, DF, ES, GO, MA, MG, } \\
\text { MS, MT, PA, PB, PR, PE, PI, } \\
\text { RJ, RO, RR, SP, TO) }\end{array}$ & $\begin{array}{l}\text { Amazônia, Caatinga, } \\
\text { Cerrado, Mata Atlântica }\end{array}$ \\
\hline *E. subrotundum A.St.-Hil. & $\begin{array}{c}\text { Corumbá: comunidade do Amolar, } \\
18^{\circ} 02^{\prime} 00^{\prime} \mathrm{S} / 57^{\circ} 29^{\prime} 60^{\prime \prime W} \text {, Borto- } \\
\text { lotto et al. } 1517 \text { (GGMS) }\end{array}$ & Sub, Arb & $\begin{array}{c}\text { Espécie restrita ao Brasil (AL, } \\
\text { BA, DF, CE, ES, GO, MG, } \\
\text { MS*, PE, RJ, RN) }\end{array}$ & $\begin{array}{c}\text { Caatinga, Cerrado, Mata } \\
\text { Atlântica }\end{array}$ \\
\hline E. tortuosum Mart. & $\begin{array}{l}\text { Três Lagoas: Amaral Jr. } 13 \\
\text { (CGMS 6874) } \\
\text { Citada por Barbosa \& Amaral Jr. } \\
\text { (2001) e Ratter } \text { et al. (2003) para } \\
\text { Mato Grosso do Sul }\end{array}$ & Arb, Arv & $\begin{array}{l}\text { Espécie sulamericana, com } \\
\text { ocorrência na Bolívia e Brasil } \\
(\mathrm{DF}, \mathrm{GO}, \mathrm{MG}, \mathrm{MS}, \mathrm{MT}, \mathrm{SP})\end{array}$ & Cerrado \\
\hline
\end{tabular}


com a lista das espécies de Erythroxylum registradas para o Mato Grosso do Sul, constatou-se que a flora desse estado possui maior similaridade com Goiás/Tocantins e São Paulo (Quadro 2). Esse fato provavelmente está relacionado à maior proximidade com ambos os estados, os quais devem estar sujeitos a fatores climáticas semelhantes.

\section{Principais grupos de pesquisa e lacunas do conhecimento}

Entre os pesquisadores que têm contribuído com vários estudos e na formação de recursos humanos (orientações de alunos de graduação e de pós-graduação) focando os representantes de Erythroxylum no Brasil, ressaltam-se os nomes de Ayrton Amaral Jr. (Universidade Estadual Paulista Júlio de Mesquita Filho/ Campus Botucatu) e Maria Iracema Bezerra Loiola (Universidade Federal do Ceará/Herbário EAC).

Atualmente, as principais lacunas de conhecimento sobre o grupo estão relacionadas especialmente às espécies da região Norte do Brasil, dada a dificuldade de coleta e deslocamento para a análise de material nos respectivos herbários. Além disso, destaca-se que são escassos os estudos direcionados à biologia reprodutiva/floral, polinizadores e filogenia das Erythroxylaceae.

\section{Principais Acervos e Perspectivas de Pesquisa para o Grupo nos próximos 10 anos}

Dentre os herbários que detêm um número considerável de amostras de Erythroxylum ocorrentes na flora brasileira, incluindo coleções-tipos, destacam-se no Brasil, os acervos dos Herbários CEPEC, IPA, INPA, RB. No exterior, ressaltam-se os acervos dos Herbários F, K, P e NY.
As floras de alguns estados nordestinos já foram concluídas ou estão em andamento. O grupo coordenado pela Profa. Maria Iracema Bezerra Loiola (UFC/Herbário EAC) está se esforçando para a conclusão da flora dos estados do Ceará e Alagoas em curto prazo. Também já foram iniciadas as análises das espécies de Erythroxylum ocorrentes nos estados do Piauí e Espírito Santo.

Destaca-se que os inventários florísticos têm contribuído para o desenvolvimento de estudos que tentam explicar a diversidade e endemismos de Erythroxylum no Brasil, relacionando os fatores ambientais e as mudanças climáticas. Trabalhos nessa linha de pesquisa estão sendo realizados junto ao Programa de Pós-graduação em Ecologia e Recursos Naturais da Universidade Federal do Ceará, como por exemplo, a dissertação intitulada "Distribuição geográfica e modelagem de nicho ecológico de espécies endêmicas de Erythroxylaceae na região Neotropical" e a tese "Diversidade filogenética de Erythroxylaceae Kunth em domínios vegetacionais brasileiros".

Todo esse conjunto de informações obtidas com as floras estaduais poderá subsidiar, num futuro próximo, a revisão das seções propostas por Schulz (1907) e a filogenia desse grupo taxonômico bem representado em nossa flora.

\section{AGRADECIMENTOS}

Aos curadores dos herbários BOTU, CGMS, ESA, F, HRCB, MBM, PEUFR, RB e UFMS pela atenção durante a consulta das coleções e/ou envio das amostras para análise; Maria Iracema Bezerra Loiola agradece ao Conselho Nacional de Desenvolvimento Científico e Tecnológico pela bolsa de pesquisador concedida.

Quadro 2. Comparação da lista das espécies de Erythroxylum registradas para Mato Grosso do Sul e os demais estados brasileiros. Acre = AC (Loiola 2008); Ceará = CE (Cordeiro 2010); Distrito Federal = DF (Mendonça et al. 2002a); Goiás/Tocantins = GO/TO (Barbosa e Amaral Jr. 2001); Paraíba = PB (Loiola et al. 2007); Paraná = PR (Mendonça et al. 1998); Rio Grande do Sul = RS (Sobral 1987); Santa Catarina = SC (Amaral Jr. 1980); Sergipe = SE (Costa-Lima et al. 2013b); (São Paulo = SP (Mendonça e Amaral Jr. 2002b).

\begin{tabular}{|c|c|c|c|c|c|c|c|c|c|c|}
\hline Espécies de Erythroxylum & $\mathrm{AC}$ & $\mathrm{CE}$ & DF & $\begin{array}{l}\mathrm{GO} / \\
\mathrm{TO}\end{array}$ & PB & PR & RS & $\mathrm{SC}$ & SE & SP \\
\hline E. anguifugum Mart. & & & & $\mathrm{x}$ & & $\mathrm{x}$ & & & & $\mathrm{x}$ \\
\hline E. campestre A.St.-Hil. & & $\mathrm{x}$ & $\mathrm{x}$ & $\mathrm{x}$ & & $\mathrm{x}$ & & & & $\mathrm{x}$ \\
\hline E. citrifolium A.St.-Hil. & $\mathrm{x}$ & $\mathrm{x}$ & & $\mathrm{x}$ & $\mathrm{x}$ & & & & $\mathrm{x}$ & $\mathrm{x}$ \\
\hline E. cuneifolium (Mart.) O.E.Schulz & & & & $\mathrm{x}$ & & $\mathrm{x}$ & $\mathrm{x}$ & $\mathrm{x}$ & & $\mathrm{x}$ \\
\hline E. daphnites Mart. & & & $\mathrm{x}$ & $\mathrm{x}$ & & & & & & $\mathrm{x}$ \\
\hline E. deciduum A.St.-Hil. & & $\mathrm{x}$ & $\mathrm{x}$ & $\mathrm{x}$ & & $\mathrm{x}$ & $\mathrm{x}$ & $\mathrm{x}$ & & $\mathrm{x}$ \\
\hline E. engleri O.E.Schulz & & & $\mathrm{x}$ & $\mathrm{x}$ & & & & & & \\
\hline E. pelleterianum A.St.-Hil. & & & & $\mathrm{x}$ & & $\mathrm{x}$ & $\mathrm{x}$ & & & $\mathrm{x}$ \\
\hline E. suberosum A.St.-Hil. & & $\mathrm{x}$ & $\mathrm{x}$ & $\mathrm{x}$ & & $\mathrm{x}$ & & & & $\mathrm{x}$ \\
\hline E. subrotundum A.St.-Hil. & & $\mathrm{x}$ & $\mathrm{x}$ & $\mathrm{x}$ & & & & & & \\
\hline E. tortuosum Mart. & & & $\mathrm{x}$ & $\mathrm{x}$ & & & & & & $\mathrm{x}$ \\
\hline
\end{tabular}




\section{REFERÊNCIAS}

Amaral Jr., A. 1973. O gênero Erythroxylum no município de Botucatu, SP. Tese 154f., Faculdade de Ciências Médicas e Biológicas de Botucatu,Universidade Estadual Paulista Júlio de Mesquita Filho, Botucatu. . 1976. Erythroxylum campinense (Erythroxylaceae), espécie nova da Amazônia. Acta Amazonica 6: 213-214. 1980. Eritroxiláceas. Flora Ilustrada Catarinense. Erythroxylaceae. 63 p.

Barbosa, A.V.G. \& Amaral Jr., A. 2001. Flora dos estados de Goiás e Tocantins: Erythroxylaceae. Coleção Rizzo 29: 9-73.

Brummitt, R.K. \& Powell, C.E. 1992. Authors of plant names. Royal Botanic Garden, Kew.732p.

Cain, S.A. \& Castro, G.M.O. 1959. Manual of vegetation analysis. Hafner, New York. 325p

Cordeiro, L.S. 2010. Flora do Ceará: Erythroxylaceae Kunth. Monografia 67f., Universidade Federal do Ceará, Fortaleza.

Costa-Lima, J.L. \& Alves, M. 2013a. A new species of Erythroxylum (Erythroxylaceae) from the Brazilian Atlantic Forest. Phytotaxa 141: 55-60.

Costa-Lima, J. L., Loiola, M.I.B., Cordeiro, L.S. \& Alves, M. 2013b. Flora de Sergipe: Erythroxylaceae. In Flora de Sergipe. (A.P.N. Prata et al., eds.). Gráfica e Editora Triunfo, Aracaju, p. 233-247.

Costa-Lima, J.L., Loiola, M.I.B. \& Alves, M. 2014a. A new species of Erythroxylum (Erythroxylaceae) from northeastern Brazil. Brittonia 66: 60-64.

Costa-Lima, J.L., Loiola, M.I.B. \& Jardim, J.G. 2014b. Erythroxylaceae no Rio Grande do Norte, Brasil. Rodriguésia 65: 659-671.

Costa-Lima, J.L. \& Alves, M. 2015a. Three new species in Brazilian Erythroxylum (Erythroxylaceae). Phytotaxa192: 97-104.

Costa-Lima, J.L. \& Alves, M. 2015b. Flora da Usina São José, Igarassu, Pernambuco: Erythroxylaceae. Rodriguésia 66: 285-295.

Centro de Referência em Informação Ambiental - CRIA 2012. SpeciesLink. Disponível em: http://www.splink.org.br/index. Acessado em 27.08.2015.

Daly, D. 2004. Erythroxylaceae. In Flowering Plants of Neotropics (N. Smith et al., eds.). Princeton University Press, The New York Botanical Garden, p. 143-145.

De Candolle, A.P. 1824. Erythroxyleae. Prodromus. 1: 537-576.

Dubs, B. 1998. Erythroxylaceae. Prodromus Florae matogrossensis. Part. I: Checklist of Angiosperms. Série B, n. 3, p. 94-97.

Loiola, M.I.B. 2001. Revisão taxonômica de Erythroxylum P. Browne sect. Rhabdophyllum O.E.Schulz (Erythroxylaceae Kunth). Tese 238f., Universidade Federal Rural de Pernambuco, Recife.

. 2004. Flora de Grão-Mogol, Minas Gerais: Erythroxylaceae. Boletim de Botânica 22(2): 101-108.

2008. Erythroxylaceae. In Primeiro Catálogo da Flora do Acre (D.C. Daly, \& M. Silveira, eds.). Universidade Federal do Acre, The New York Botanical Garden, Rio Branco, p. 190-191.

2013. A new species of Erythroxylum (Erythroxylaceae) from the Brazilian semiarid region. Phytotaxa 150:61-64.

Loiola, M.I.B., Sales, M.F., Mayo, S.J. \& Rodal, M.J.N. 1998. Erythroxylaceae. In Plantas Vasculares das Florestas Serranas de Pernambuco. Um checklist da flora ameaçada dos brejos de altitude. Pernambuco-Brasil. (F.A. Juncá, ed.). Universidade Federal Rural de Pernambuco, Recife, Pernambuco, p. 61-61.

Loiola, M.I.B., Agra, M.F., Baracho, G.S. \& Queiroz, R.T. 2007. Flora da Paraíba, Brasil: Erythroxylaceae Kunth. Acta Botanica Brasilica 21: 473-487.
Loiola, M.I.B. \& Sales, M.F. 2008. Two new species of Erythroxylum sect. Rhabdophyllum (Erythroxylaceae) from north-eastern Brazil. Kew Bulletin 63: 655-659.

Loiola, M.I.B. \& Gomes, J.M.O. 2009. Flora de Mirandiba: Erythroxylaceae. In Flora de Mirandiba. (M. Alves et al., org.).1ed. Recife: Associação Plantas do Nordeste, v.1, p. 146-148.

Loiola, M.I.B. \& Sales, M.F. 2012. Erythroxylum ayrtonianum (Erythroxylaceae): a new species from Brazil. Novon 22: 48-50.

Loiola, M.I.B \& Cordeiro, L.S. 2014. Erythroxylum sobraleanum (Erythroxylaceae): a new species from Southeastern Brazil. Phytotaxa 183: 56-60.

Loiola, M.I.B., Costa-Lima, J.L., Mayo, S.J., Sales, M.F. 2014. Nomenclatural notes on Erythroxylum sect. Rhabdophyllum (Erythroxylaceae): new synonyms and lectotypifications. Nordic Journal of Botany 33: 451-454.

Loiola, M.I.B. \& Costa-Lima, J.L. 2015. Erythroxylaceae. In Lista de Espécies da Flora do Brasil. Disponível em: http://floradobrasil.jbrj. gov.br/2010/FB000111. Acessado em 27.08.2015.

Mabberley, D.J. 1990. The Plant-Book: A portable dictionary of the higher plants. University Press, Cambridge. 706p.

Martius, K.F.P. 1840. Beiträge zur Kenntniss de Gattung Erythroxylon. 2: $1-130$.

.1843. Erythroxylon. Abhandlungen der Königlichen Bayerischen Akademie der Wissenschaften 3(2): 280-410.

Mendonça, J.O., Cervi, A.C. \& Guimarães, O.A. 1998. O gênero Erthroxylum P. Browne (Erythroxylaceae) do Estado do Paraná, Brasil. Brazilian Archives of Biology and Technology 41:349-358.

Mendonça, J.O. \& Amaral Jr., A. 2002a. Erythroxylaceae. In Flora do Distrito Federal (Cavalcanti, T.B. \& Ramos, A.E., orgs.). Embrapa Recursos Genéticos e Biotecnologia, Brasília, v. 2, p. 53-72.

.2002b. Erythroxylaceae. In Flora Fanerogâmica do Estado de São Paulo (M.G.L. Wanderley et al., eds). FAPESP, HUCITEC, São Paulo, p. 107-119.

Peyristsch, J. 1878. Erythroxylaceae. In Flora Brasiliensis (C.F.P. Martius, ed.). F. Fleischer, Lipsiae, v. 12, pars 1, p. 125-180.

Plowman, T. 1983. New species of Erythroxylum from Brazil and Venezuela. Botanical Museum Leaflets 29: 273-290. .1984. New taxa of Erythroxylum (Erythroxylaceae) from the Amazon Basin. Acta Amazonica. Supl. 14: 117-143.

.1986. Four new species of Erythroxylum (Erythroxylaceae) from northeastern Brazil. Brittonia 38: 189-200.

. 1987. Ten new species of Erythroxylum (Erythroxylaceae) from Bahia, Brazil. Fieldiana: Bot. 19: 1-41.

Plowman, T. \& Berry, P.E. 1999. Flora of Venezuelana Guayana. 5: 59-71.

Plowman, T. \& Hensold, N. 2004. Names, types and distribution of neotropical species of Erythroxylum (Erythroxylaceae). Brittonia 56: $1-53$.

Ratter, J.A., Bridgewater, S., Ribeiro, J.F., Fonsêca-Filho, J., Da Silva, M.R., Milliken, W., Pullan, M., Pott, A., Oliveira-Filho, A., Durigan, G. \& Pennington, R.T. 2003. Analysis of the floristic composition of the Brazilian cerrado vegetation IV: Presentation of a Revised Data-Base of 367 Areas. Disponível em: http://cerrado.rbge.org.uk/ cerrado/download/download.php. Acessado em 28.8.2015.

Schulz, O.E. 1907. Erythroxylaceae. In Das Pflanzenreich (A. G. Engler, ed.). Verlag von Wilhelm Engelman, Leipzig,v. 4, p. 1-164.

Sobral, M. 1987. Erythroxylum (Erythroxylaceae) no Rio Grande do Sul. Cadernos de Pesquisa, Série Botânica 38: 7-42.

Whittaker, R.H. 1975. Communities and ecosystems. Collier-Macmillan Canada Ltd., New York. 385p. 